\title{
LXIII. A kinetic theory of solids, with an experimental introduction
}

\section{William Sutherland}

To cite this article: William Sutherland (1891) LXIII. A kinetic theory of solids, with an experimental introduction , Philosophical Magazine Series 5, 32:199, 524-553, DOI:

10.1080/14786449108620220

To link to this article: http://dx.doi.org/10.1080/14786449108620220

曲 Published online: 08 May 2009.

Submit your article to this journal $\lceil\pi$

Џ Article views: 7

Q View related articles $\asymp$ 


\section{$\left[\begin{array}{ll}524 & ]\end{array}\right.$}

\section{A Kinetic Theory of Solids, with an Experimental Introduction. By William SutherLand.}

[Concluded from p. 225.]

\section{THEORY.}

TTHE working out of a kinetic theory of solids ought, on 1 purely theoretic grounds, to have proved a simpler matter than that of the kinetic theory of gases, because in solids each molecule abides about a fixed mean position; but the generality of the two great experimental laws of perfect gases stimulated theory to overcome the more difficult task first. The experimental laws which have just been established for solids seem to give as good a guarantee that in its essence the kinetic theory of solids must be as simple a matter as that of gases.

We will therefore start out on the assumption that in solids the molecules have the properties assigned to them in the kinetic theory of gases, that they attract one another, and that each one moves in a small region of space round a certain mean position. In the almost purely statical molecular theories of solids put forth in the early part of this century by the great French elasticians, repulsive forces had to be imagined to equilibrate the attractive; for example, Poisson's molecular theory was founded on the supposition that while molecules of matter attracted molecules of matter, they repelled the particles of heat, so that each molecule was in equilibrium under two sets of opposite central forces. But it is easy to see that no theory founded on purely central attractions and repulsions can explain the facts of rigidity as we actually know them, because in a pure shear, as there is no change of volume, there can be no change in the potential energy of the purely centrally acting molecules; or, more accurately, a small shear of the first order will produce a change of potential energy of the second order of small quantities, but experiment shows that the change of energy in a shear is of the same order of magnitude as the change that accompanies a change of volume: hence purely central attractions and repulsions, while they can give a sort of rigidity, give one infinitely different from that of nature. The statical theory can give equations of the same form as the natural omes, but with some of the coefficients infinitely smaller.

Attempts have been made to avoid this difficulty by giving the molecules polar properties, which, however, can hardly be 
got to be compatible with the behaviour of isotropic bodies. The true solution of the difficulty lies in taking account of the kinetics of the molecules. The motion of the molecules is a fundamental part of the phenomenon of elasticity of solids as of gases.

The subject-matter of the kinetic theory of solids will be taken in the following order :-

1. Establishment of the characteristic equations of solids.

2. Testing of the equations on the supposition that the molecules are unalterable.

3. Testing of equations on the supposition that the molecules alter somewhat with change of temperature.

4. Further evidence of the alteration of molecules with change of temperature.

5. Periods of vibration of the molecules of metals.

6. Comparison of the theoretical variation of Young's modulus with temperature with the experimental.

7. Rigidity according to the kinetic theory.

8. Ratio of lateral contraction to elongation in Young's experiment.

9. Dulong and Petit's law, Joule and Kopp's law, and thie equation for compound solids.

10: The parameter of molecular force.

1. Establishment of the characteristic Equations of Solids.In the kinetic theory of gases molecules are supposed to exercise pressure on an external body by colliding against its molecules, and the molecules are supposed to collide with one another. Let us imagine, then, a number of molecules of equal mass and size moving amongst one another under the influence of an attractive force $\phi(r)$ exerted by each, and colliding and rebounding with coefficient of restitution unity. If the molectles are close enough together, it is evident that each separate molecule is hemmed in by its neighbours, so that it cannot change its position beyond vibrating about in its own limited share of space which I call its domain. Such a collection of molecules has one characteristic property of a solid body. Liquefaction occurs when each molecule ceases to be hemmed in and just manages to wriggle through amongst its neighbours. In the solid body each molecule as it knocks about in its domain has its mean position preserved, just as if it were held there by central repulsive forces emanating from all the other molecules, or only from its immediate neighbours, while it itself by colliding with its neighbours many times a second in all directions acts virtually as a centre of repulsion to them. There is this much 
in common, then, between the kinetic theory and the old static ones, with the great difference, however, that the kinetic state of the molecules is taken account of and regarded as the fundamental cause of the effective repulsions, and these repulsions act only between immediate neighbours, and do not extend to all distances as the attractions do.

What are the conditions of equilibrium in our system of molecules? First, let us take the case of a solid homogeneous and isotropic body free from external force. Suppose it divided into two parts by a plane, and on unit area in the plane suppose a right cylinder erected in one part of the body ; this cylinder as a whole is attracted by the half of the body on the other side of the plane, and this attraction can be equilibrated only by the repulsion due to the collisions occurring across the base of the cylinder. Accordingly we must evaluate these two forces. Let the distribution of molecules be a cubical one with edge of cube $e$; then, in the manner of Poisson (Journal de l'Ecole Polytech. Cahier xx.), the attraction on the cylinder is easily proved to be $\frac{1}{6 e^{3}} \Sigma r \phi(r)$, where the summation extends to all molecules within reach of appreciable action of one fixed molecule.

To evaluate the collisional repulsion on the base of the cylinder, we will first take a short cut justified by the theory of gases, and then verify the result by another method. The short cut consists in assuming that the collisional transfer of momentum goes on as if all the molecules had the average velocity square, and as if one third of the number always travelled parallel to each of three rectangular axes. Take one axis to be perpendicular to the unit-base of the cylinder, and let $n$ be the number of molecules per unit area, so that $n=1 / e^{2}$, then $n / 3$ molecules collide against the base of the cylinder with velocity $v$, and the number of times per second that each strikes the base is $v / 2 \alpha$, where $\alpha$ is the mean swing of a molecule inside its domain. As each molecule has its velocity reversed by a collision, the force of the collisions on the base of the cylinder is $\frac{n}{3} 2 m v \frac{v}{2 \alpha}$ or $m v^{2} / 3 e^{2} \alpha$, and for equilibrium we have the equation

$$
\frac{m v^{2}}{3 e^{2} \alpha}-\frac{1}{6 e^{3}} \Sigma r \phi(r)=0 ; . . . . .
$$

or, as we have been dealing with average values, we may as well sum for all the molecules of the body and put our result in the form

$$
\Sigma \frac{m v^{2}}{2 \alpha}-\frac{1}{2} \cdot \frac{1}{2} \cdot \frac{1}{e} \Sigma \Sigma r \phi(r)=0 . \quad . \quad .
$$


The same equation will now be established from Clausius's equation of the virial, taking account of the virial of the repulsions during collision as Lorentz does (Wied. Ann. xii.) in the case of gases. Let $\mathrm{F}$ be the repulsive force between two molecules in collision when their centres are distance $\mathbf{E}$ apart, then the equation of the virial is

$$
\begin{gathered}
\Sigma \frac{1}{t} \int_{0}^{t} \frac{m v^{2}}{2} d t \\
=\frac{1}{2} \cdot \frac{1}{2} \Sigma \Sigma \frac{1}{t} \int_{0}^{t} r \phi(r) d t-\frac{1}{2} \cdot \frac{1}{2} \Sigma \frac{1}{t} \int_{0}^{t} \mathrm{EF} d t+\text { terms }
\end{gathered}
$$

which vanish when the motion is stationary and $t$ is large enough. As $\mathrm{E}$ is practically constant during collision,

$$
\int \mathrm{EF} d t=\mathrm{E} \int \mathrm{F} d t \text {. }
$$

Now suppose each encounter to last a time $\tau$, then, since each molecule in time $t$ experiences $v t / a$ collisions, we have

and then

$$
\int_{0}^{t} \mathrm{EF} d t=\frac{v t \mathrm{E}}{\alpha} \int_{0}^{\tau} \mathrm{F} d t=\frac{v t \mathrm{E}}{\alpha} 2 m v ;
$$

$$
\begin{gathered}
\Sigma \frac{m v^{2}}{2}=\frac{1}{2} \cdot \frac{1}{2} \Sigma \Sigma r \phi(r)-\frac{1}{2} \cdot \frac{1}{2} \Sigma \frac{2 m v^{2} \mathrm{E}}{\alpha}, \\
\therefore \Sigma \frac{m v^{2}}{2}\left(1+\frac{\mathrm{E}}{\alpha}\right)=\frac{1}{2} \cdot \frac{1}{2} \Sigma \Sigma r \phi(r) .
\end{gathered}
$$

Now $\mathrm{E}$ is the mean distance apart of the molecules when in contact, and accordingly

$$
\mathrm{E}+\alpha=e ;
$$

hence, as before,

$$
\frac{1}{\alpha} \Sigma \frac{m v^{2}}{2}-\frac{1}{2} \cdot \frac{1}{2} \cdot \frac{1}{e} \Sigma \Sigma r \phi(r)=0 .
$$

In the usual theory of gases the molecules are treated as elastic spheres, in which case $\mathrm{E}$ is constant; but as change of temperature is known to produce changes inside the molecule, it will be safer to consider $E$ as possibly a function of the temperature ; E can hardly depend appreciably on the pressure, except in cases where, by the application of external pressure, the number of collisions per second becomes great enough to influence the internal structure of the molecule, as, for example, when great pressure produces crystallizations and combinations. In the most general case, then, we have 
to consider $\mathrm{E}$ as possibly a function of both temperature and pressure. But we can first see how the supposition that $\mathrm{E}$ is constant will work. The equation (1) then states that, given E the size of the molecules, their total energy, and their law of force, then $e$, the mean distance apart of the molecules free from external force, is determinable. The equation specifies the law of expansion of the simplest solid free of external stress. For example, imagine that we know only that solids expand very slightly with rising temperature, then $\frac{1}{e} \Sigma \Sigma, r \phi(r)$ is probably nearly constant, and therefore a must be nearly proportional to the kinetic energy, or the expansion from absolute zero is nearly proportional to the absolute temperature, which is the definite experimental result. But before discussing this equation any further, we may as well make it more general by taking account of external stress and removing the condition of isotropy.

Imagine the properties of our molecules to be different in different directions, and in such a manner that all the circumstances can be specified with reference to three rectangular axes. Let the paths and relocities of the molecules be such that they produce the same collisional pressure in the direction of the $x$ axis as if they were an isotropic system with mean kinetic energy $m u^{2} / 2$ per molecule, and similarly kinetic energy $m v^{2} / 2$ and $m w^{2} / 2$ along the $y$ and $z$ axes, so that $\left(m u^{2}+m v^{2}+m w^{2}\right) / 2=3 \mathrm{D}$, where $\mathrm{D}$ is the mean kinetic energy per molecule of the actual system. Let the mean positions of the molecules correspond to a parallelepiped distribution with sides $\xi, \eta, \zeta$ to the parallelepiped, so that the mean distance apart of the molecules along the $x$ axis is $\xi$, and so on. Let the external stress be specified by pressures $\mathrm{P}, \mathrm{Q}, \mathrm{R}$ parallel to the three axes, and let the mean components of the swing of each molecule in its domain be $\alpha, \beta, \gamma$, then for equilibrium parallel to the $x$ axis we have merely to add $P$ to the molecular pressure in equation (1), and make the necessary slight alterations to get

$$
\frac{m u^{2}}{3 \eta \xi_{\alpha}}-\mathrm{P}-\frac{1}{6 \xi \eta \zeta} \Sigma r \phi(r)=0, . . . .
$$

with two similar equations.

If $\exists \mathbf{H Z}$ are the values of $\xi, \eta, \zeta$ when the molecules are in contact, we have three equations of the form $\xi-\Xi=\alpha$. Hence, given $\Xi, \mathrm{H}, \mathrm{Z}, u^{2}, v^{2}, w^{2}, \mathrm{P}, \mathrm{Q}, \mathrm{R}$, and the law of force, the problem of finding $\xi, \eta$, $\zeta$ is determinate ; that is, the law of expansion in the three directions with increase of kinetic 
energy under a given stress. But if the separate components $u^{2}, v^{2}, w w^{2}$ are not given, but only $\mathrm{D}$ the mean kinetic energy, then, given $P, Q, R, D$, that is to say the stress and the temperature as well as $\Xi, \mathbf{H}, \mathbf{Z}$, there are four relations connecting the six quantities $u^{2}, v^{2}, w^{2}, \xi, \eta, \zeta$. Two other relations would make the connexion between energy and size in different directions quite determinate ; it is the business of experiment to ascertain these two relations.

If the body is homogeneous and isotropic when free from external stress, then the equations (2) are a little simplified by the relation $\Xi=\mathrm{H}=\mathrm{Z}=\mathrm{E}$, and we have

$$
\frac{r_{m u^{2}}}{3 \eta \zeta(\xi-\epsilon)}-\mathrm{P}-\frac{1}{6 \xi \eta \zeta} \Sigma r \phi(r)=0, \ldots .
$$

with two similar equations.

If, further, the body is subjected to hydrostatic pressure $p$ only, so that $\mathrm{P}=\mathrm{Q}=\mathrm{R}=p$, then the three equations of form (3) reduce to the one

$$
\frac{2 \mathrm{D}}{3 e^{2}(e-\mathrm{E})}-p-\frac{1}{6 e^{3}} \Sigma r \phi(r)=0 . \quad . \quad .
$$

Thus, if the size of the molecules is given, and also the law of force, then the relation of pressure, volume, and kinetic energy is determinate. Accordingly, we are able to calculate the bulk-modulus of the solid (the reciprocal of its compressibility).

Let $\omega$ denote volume, then the bulk-modulus is $\omega d p / d \omega$; denoted by

$$
k=\omega d p / d \omega=\frac{2}{3} \omega \frac{d}{d \omega}\left\{\frac{\mathrm{D}}{e^{2}(e-\mathrm{E})}-\frac{1}{4 e^{3}} \Sigma r \phi(r)\right\} .
$$

But $\omega=\nu e^{3}$, where $\nu$ is the number of molecules per unit volume, and so

$$
k=\frac{2}{3} \cdot \frac{e}{3} \frac{d}{d e}\left\{\frac{\mathrm{D}}{e^{2}(e-\mathrm{E})}-\frac{1}{4 e^{3}} \Sigma r \phi(r)\right\} . .
$$

We can go no further without the law of molecular force, so I will now use the law of force, which I have formerly discussed in connexion with gases and liquids (Phil. Mag. 5th ser. xxiv. \& xxvii.), namely, $\phi(r)=3 \mathrm{~A} m^{2} / r^{4}$. Then

$$
\Sigma r \phi(r)=\int_{a}^{\mathrm{R}} \frac{4 \pi r^{2} d r 3 \mathrm{~A} m \rho r}{r^{4}}
$$

Phil. Mag. S. 5. Vol. 32. No. 199. Dec. 1891. $\quad 2 \mathrm{~N}$ 
where $a$ is a length of the order of $e$, and $\mathrm{R}$ is a length of the order of the linear dimensions of the body.

$$
\Sigma r \phi(r)=12 \mathrm{~A} m \pi \rho \log \mathrm{R} / a \text {. }
$$

On account of the smallness of $a, \log \mathbf{R} / a$ is approximately the same for all ordinary bodies, and then $\Sigma r \phi(r)$ for a given body is always proportional to the density, and for different bodies its value depends on that of a parameter $A$, which has a definite value for each body. Apart from all specification of the law of molecular force, if the potential energy and the virial of a number of molecules are proportional to the density of their distribution, and can be represented by such forms as $B \rho$, where $B$ is constant for each substance, the same conclusions will hold as those I am about to draw from the law of the inverse fourth power. My previous arguments in favour of the inverse fourth power law amounted to this, that that is the only simple natural law, like the law of gravitation, that will make the potential energy of a number of molecules and their virial both closely proportional to the density of distribution, as experiment shows they must be.

With the.above value of $\Sigma r \phi(r)$ and with $\rho=m / e^{3}$, we have

$$
\begin{aligned}
k & =\frac{2}{3} \frac{e}{3} \frac{d}{d e}\left\{\frac{\mathrm{D}}{e^{2}(e-\mathrm{E})}-\frac{3 \mathrm{~A} m^{2} \pi \log \mathrm{R} / a}{e^{6}}\right\} \\
& =\frac{2}{3} \frac{e}{3}\left\{-\frac{2 \mathrm{D}}{e^{3}(e-\mathrm{E})}-\frac{\mathrm{D}}{e^{2}(e-\mathrm{E})^{2}}+\frac{18 \mathrm{~A} m^{2} \pi \log \mathrm{R} / a}{e^{7}}\right\} .
\end{aligned}
$$

But in the absence of external stress (4) becomes

and hence

$$
\frac{\mathrm{D}}{e^{2}(e-\mathrm{E})}=\frac{3 \mathrm{~A} m^{2} \pi \log \mathrm{R} / a}{e^{6}},
$$

$$
k=\frac{2}{3} \frac{e}{3}\left\{\frac{4 \mathrm{D}}{e^{3}(e-\mathrm{E})}-\frac{\mathrm{D}}{e^{2}(e-\mathrm{E})^{2}}\right\} . \quad .
$$

2. Testing of the Equations on the supposition that the Molecules are unalterable.-Suppose that $\mathrm{E}$ is constant, then if $b$ is the mean coefficient of linear expansion between zero and $\theta$ which corresponds to kinetic energy $D$, then $(e-\mathrm{E}) / e=b \theta$, and if $c$ is the mean specific heat between zero and $\theta$, then $\mathrm{D}=\mathrm{J}_{m c} \theta$, where $J$ is the mechanical equivalent of heat, if we suppose that all the energy of the molecules exists in their vibrations as wholes; if not, then we must write $\mathrm{D}=h \mathrm{~J} \operatorname{cm} \theta$, where $h$ is a fraction probably not differing much from unity. Thus we have

$$
k=\frac{2}{3} h \cdot \frac{\mathrm{J} c m}{3 b m / p}\left(4-\frac{1}{b \theta}\right) . . . . .
$$


Even at the melting-point $1 / 6 \theta$ for most metals is about 50 , so that at ordinary temperatures 4 is small in comparison with $1 / b \theta$. Neglecting 4 for the present, and remembering that $c$ and $b$ vary only to a small extent with temperature, and that $\mathrm{cm}$, by Dulong and Petit's law, is the same for all metals, we can write, putting $h=1$,

$$
k=-\frac{2}{9} \frac{J_{c m}}{b^{2} \theta m / \rho} \cdot \cdot . \cdot . \quad . \quad . \quad .
$$

approximately, and assert that theoretically the bulk-modulus varies inversely as the temperature, and that the product $k l^{2} \theta \mathrm{m} / \rho$ is the same for all metals. This result makes the bulk-modulus infinite at absolute zero, which, of course, is merely the result of our assumption that the change of volume of the molecules produced by collisions is negligible. At any rate the bulk-modulus at absolnte zero is probably very large, that is, the molecules are probably only slightly compressible, as will be seen later on.

In our equations (7) and (8) for $k$ we know all the quantities on the right-hand side (if $h=1$ ) for the metals, and we also have some quasi-experimental determinations of $k$, so that we ean proceed to test how our assumption of $\mathrm{E}$ being constant will hold. The values of $k$ are not purely experimental, because they are not obtained by direct measurement, but by calculations from other elastic measurements on the assumption that the metals are homogeneous isotropic solids. In such solids $k$ is connected with the rigidity $n$, and Young's modulus $q$ by the relation $k=n q /(9 n-3 q)$.

When $k$ is calculated from the experimental values of $n$ and $q$, the worth of the result depends entirely on the nearness to perfect isotropy of the solid. Thus, on the assumption of perfect isotropy, it is possible to calculate a value of $k$ for many metals at $15^{\circ} \mathrm{C}$. from the average values of $n$ and $q$ given above in Tables III. and VII. Tomlinson has given (Phil. Trans. 1883) values of $k$ calculated from his measurements of $n$ and $q$; and there is this advantage in using his values, that $n$ and $q$ were measured on the same specimen. Amagat has found (Compt. Rend. cviii.) values of $k$ for a few bodies by measuring $q$, and also the change of volume produced by external pressure on hollow cylinders, and then calculating on the assumption of perfect isotropy. It will be shown afterwards that the metals are at least nearly isotropic, but yet we must assume that all our values of $k$ for the metals are only more or less rough approximations to true values, because small departures from perfect isotropy produce large 
errors in the value of $k$ given by the formula for perfect isotropy.

The following Table contains in the row $k_{1}$ the values of $k$ calculated from my average values of $n$ and $q$ given in Tables III. and VII., in $k_{2}$ Tomlinson's valnes, in $k_{3}$ Amagat's, and in $k_{4}$ the values found from equation (7) above, using Fizeau's values of $b$. The temperature is $15^{\circ} \mathrm{C}$. in all. In the last row are given the values of the ratio of $k_{4}$ to the mean of $k_{1}, k_{2}$, and $k_{3}$.

\begin{tabular}{|c|c|c|c|c|c|c|c|c|c|}
\hline $\begin{array}{r}\mathrm{Cu} . \\
10^{-6} k_{1} \ldots 2500\end{array}$ & $\begin{array}{l}\text { Ag. } \\
690\end{array}$ & $\begin{array}{c}\text { Au. } \\
1370\end{array}$ & $\begin{array}{l}\mathrm{Mg} . \\
325\end{array}$ & $\begin{array}{l}\mathrm{Zn} . \\
900\end{array}$ & $\begin{array}{l}\text { Al. } \\
810\end{array}$ & $\begin{array}{l}\text { Sn. } \\
\text { imp. }\end{array}$ & $\begin{array}{r}\mathrm{Pb} . \\
86\end{array}$ & $\begin{array}{c}\mathrm{Fe} \\
2000\end{array}$ & $\begin{array}{l}\text { Pt. } \\
990\end{array}$ \\
\hline $10^{-6} k_{2} \ldots 980$ & 930 & $\ldots$ & $\cdots$ & 350 & 320 & 130 & 76 & 1500 & \\
\hline $10^{-6} k_{3} \ldots 1170$ & $\ldots$ & $\cdots$ & $*$ & $\ldots$ & $\cdots$ & $\cdots$ & 360 & 1470 & \\
\hline $10^{-8} k_{4} \ldots \quad 970$ & 520 & 980 & 190 & 250 & 325 & 264 & 134 & 2000 & \\
\hline Ratio ... & 64 & 71 & 59 & 40 & 58 & 203 & 77 & 120 & \\
\hline
\end{tabular}

There are large discrepancies in the quasi-experimental values of $k$; for example, in the case of tin $k_{1}$ is impossible, because $9 n-3 q$ is negative; accordingly tin is so far from being reliably isotropic as to make attempts at calculating $k$ illusory. If, besides tin, we reject platinum, we find fairly good agreement amongst the values of the ratio of $k_{4}$, the theoretical value, to the mean quasi-experimental value. The mean value of the ratio, leaving out iron, is 62 , and including iron is 69 ; so that we can say that the theoretical values of the bulk-modulus of the metals, on the assumption that the molecules do not change in size with changing temperature, are about 70 times the actual values. Thus, while the assumption of constant size in molecules is proved by this comparison to be untenable, the general form of our equation receives some partial verification from it, as it shows $k b^{2} \theta m / \rho$ to be nearly the same for 8 out of 9 metals, and this is what the form of our equation demanded.

But fortunately there is a more satisfactory comparison to be made between theory and experiment in the matter of latent heats of melting. We cannot imagine our equation to bridge the gap in continuity between the solid and liquid states and caleulate the latent heat by the formula

$$
\lambda=\int_{v_{1}}^{v_{2}} \theta \partial \rho / \partial \theta d v
$$

where $v_{2}$ and $v_{1}$ are the volumes' of unit mass in the liquid and solid states. But without any bridging of the gap in continuity we have the thermodynamic relation

$$
\lambda=\left(v_{2}-v_{1}\right) \theta d p / d \theta,
$$


where $d p / d \theta$ means now the rate of increase of the meltingpressure with the melting-temperature. Now, according to our theory, melting occurs when molecules are able to escape from imprisonment by their neigbbours, that is, when the space between molecules attains a certain value relatively to the size of the molecules. Hence, if the size of the molecules is invariable, melting must always occur for the same value of $e$ whatever the pressure is, and we have $d p / d \theta$ of the last formula the same as $\partial p / \partial \theta$ taken from our equation with the condition $e=$ constant.

$$
\frac{\partial p}{\partial \theta}=\frac{2 d \mathrm{D} / d \theta}{3 e^{2}(e-\mathrm{E})} \text { and } \lambda=\frac{2 \mathrm{D}\left(v_{2}-v_{1}\right)}{3 e^{2}(e-\mathrm{E})} . .
$$

But on this view of melting the latent heat may be calculated in another manner, for on this purely mechanical view it must be the energy supplied to produce the change of potential energy that accompanies the change of density on liquefaction. Hence $\lambda=B\left(\rho_{1}-\rho_{2}\right)$, and neglecting $p$ in our equation, this

$$
\begin{gathered}
=\frac{2 \mathrm{D}}{3 e^{2}(e-\mathrm{E})} \cdot \frac{\rho_{1}-\rho_{2}}{\rho_{1}{ }^{2}} ; \\
\therefore \lambda=\frac{2 \mathrm{D}\left(v_{2}-v_{1}\right)}{3 e^{2}(e-\mathrm{E})} \cdot \frac{v_{1}}{v_{2}}=\frac{2 \mathrm{D}\left(v_{2}-v_{1}\right)}{3 e^{2}(e-\mathrm{E})} \text { nearly, }
\end{gathered}
$$

as before. Hence, remembering that $e^{3}=m / \rho, \mathrm{D}=\mathrm{J} \operatorname{cm} \theta$, and $e-\mathrm{E}=e b \theta$ nearly, we can write

$$
\lambda=\frac{2 \mathrm{~cm}}{3 \mathrm{bm}} \cdot \frac{\rho_{1}-\rho_{2}}{\rho_{1}} \text { calories. . . . . }
$$

Person (Ann. de Ch. et de Ph. sér. 3, t. 24) has found the latent heats of several metals, and Vincentini and Omodei (Wied. Beibl. xii.) have found the change of volume at the instant of melting for lead, cadmium, tin, mercury, and other bodies; and Roberts and Wrightson (Nature, xxiv.), amongst other data, give the densities of melted zinc and silver, whose densities just before melting can be calculated approximately by means of their coefficients of expansion, though this is not so satisfactory as a direct measurement. The mean coefficient of expansion $b$ of solid mercury is not known, but can be found by the relation $b \mathrm{~T} m^{\frac{1}{6}}=\cdot 044$, where $\mathrm{T}$ is meltingpoint, to be afterwards discussed. Person, in his calculations, intentionally ignored the increase of specific heat with temperature, but if we put $c=c_{0}(1+\alpha t)$ and use the values of a given by Naccari (Wied. Beibl. xii.) from experiments 
between zero and $320^{\circ} \mathrm{C}$, we can get the true latent heats we require by subtracting $\frac{1}{2} c_{0} \alpha \mathrm{I}^{2}$ from Person's values. Thus we can compare our theoretical values of $\lambda$ with the experimental in the case of six metals. The following Table contains the values of $\rho_{1}$ and $\rho_{2}$, Person's value of the latent heat $\lambda_{1}$, the true latent heat $\lambda_{2}$, and the value $\lambda_{3}$ calculated on the supposition that the molecules are invariable:-

\begin{tabular}{lrrcccc} 
& Ag. & \multicolumn{1}{c}{ Zn. } & Cd. & Hg. & \multicolumn{1}{c}{ Sn. } & Pb. \\
$o_{1} \ldots \ldots \ldots \ldots$ & $9 \cdot 92$ & $6 \cdot 85$ & $8 \cdot 366$ & $14 \cdot 19$ & $7 \cdot 183$ & $11 \cdot 00$ \\
$\rho_{2} \ldots \ldots \ldots \ldots$. & $9 \cdot 51$ & $6 \cdot 48$ & $7 \cdot 99$ & $13 \cdot 69$ & $6 \cdot 99$ & $10 \cdot 64$ \\
$\lambda_{1} \ldots \ldots \ldots \ldots$. & $21 \cdot 1$ & $28 \cdot 1$ & $13 \cdot 6$ & $2 \cdot 8$ & $14 \cdot 2$ & $5 \cdot 4$ \\
$\lambda_{2} \ldots \ldots \ldots \ldots$ & $16 \cdot 2$ & $24 \cdot 5$ & $12 \cdot 5$ & $2 \cdot 8$ & $12 \cdot 8$ & $4 \cdot 8$ \\
$\lambda_{3} \ldots \ldots \ldots \ldots$ & $81 \cdot 0$ & $117 \cdot 0$ & $53 \cdot 3$ & $9 \cdot 7$ & $46 \cdot 0$ & $23 \cdot 6$ \\
Ratio $\lambda_{3} / \lambda_{2}$. & $5 \cdot 0$ & $4 \cdot 8$ & $4 \cdot 3$ & $3 \cdot 2$ & $3 \cdot 6$ & $4 \cdot 9$
\end{tabular}

In the case of silver it was not safe to extrapolate for specific heat up to the melting-point of $954^{\circ} \mathrm{C}$. from experiments up to $320^{\circ}$, so I made a determination of its mean specific heat between $20^{\circ}$ and $860^{\circ}$ in comparison with that of gold, which is known; the result was 0663 , which, over a range of $934^{\circ}$, gives $61^{\cdot 9}$ calories in place of Person's 57. In the case of tin I used Tomlinson's determination of the specific heat, as Naccari omitted it. The ratio $\lambda_{3} \lambda_{2}$ comes out nearly constant, especially if we omit mercury as uncertain on account of the smallness of $\lambda$, and tin as uncertain on account of its tendency to crystallize; the mean value of the ratio for the other four metals is 4.75 . In each case the calculated value of $\lambda$ is about 4.75 times the experimental, and here again, while the actual equation and the assumption of inalterability in the molecules are not verified, the form of the equation is confirmed in a striking manner if we remember that the latent heat ranges from $2 \cdot 8$ for nercury to 28 for zinc. In other words equation (10) asserts that

$$
2 c\left(\rho_{1}-\rho_{2}\right) / 3 b \rho_{1} \lambda
$$

is constant on purely theoretical grounds, and the tabulated comparison shows it to be constant, only that the value of the constant is, on the average, $4 \cdot 75$ instead of 1 ; still it is promising confirmation for the kinetic theory that it should be constant at all.

We may state, then, that collections of unaltering molecules, having the coefficients of expansion of the different metals, would, on the kinetic theory, have bulk-moduluses 
about 65 times as large as the actual, and latent heats about 4.75 times as large.

3. Testing of Equations on the supposition that the Molecules alter somewhat with Change of Temperature.-The next step is to ascertain what modification in the assumptions so far made would bring theory and experiment into accord. If we return to the approximate expressions

and

$$
\text { (6) } k=-2 \mathrm{D} / 9 e(e-\mathrm{E})^{2}
$$

$$
\text { (9) } \lambda=2 \mathrm{D}\left(v_{2}-v_{1}\right) / 3 e^{2}(e-\mathbf{E}) \text {, }
$$

we see at once that if $\mathbf{E}$, which represents the linear dimensions of the molecule, were to diminish with rising temperature, then theory and experiment could be brought to better accord. Now the shrinking of molecules with rising temperature is a result required by the kinetic theory of gases, for the variation of the viscosity of gases with temperature is such as can be accounted for satisfactorily only by shrinkage of molecules with rising temperature.

Holman (Phil. Mag. 5th ser. vol. xxi.) has made a thorough comparison of the results of all the best measurements of the effect of temperature on the viscosity of air, including his own, and his series of curves shows that up to $250^{\circ} \mathrm{C}$. the viscosity of air at any temperature is best given in terms of that at zero $\mathrm{C}$. by the exponential formula

$$
\eta_{t}=\eta_{0}(1+0.00367 t)^{0.76} \text { or } \eta_{\theta}=\eta_{273}(\theta / 273)^{0.6} \text {, }
$$

where $\theta$ is absolute temperature. Now, according to the kinetic theory of gases, assuming the molecules to be spheres of sectional area $\sigma_{\theta}{ }^{2}$, and ignoring molecular attraction, we have

and hence

$$
\eta_{\theta}=\eta_{273}\left(\sigma_{273} / \sigma_{\theta}\right)^{2}(\theta / 273)^{\frac{1}{2}},
$$

$$
\left(\sigma_{273} / \sigma_{\theta}\right)^{2}=(\theta / 273)^{0.26} ;
$$

that is to say, the molecules shrink with rising temperature. Now Barus (Amer. Journ. Sci. 3rd ser. vol. cxxxv.) has given weighty support to this idea of molecular shrinking by pushing the study of the viscosity of air and hydrogen up to $1200^{\circ} \mathrm{C}$., and showing that up to that temperature the viscosity of both gases is well represented by

according to which

$$
\eta_{\theta}=\eta_{273}(\theta / 273)^{\frac{2}{3}} \text {, }
$$

$$
\left(\sigma_{273} / \sigma_{\theta}\right)^{2}=(\theta / 273)^{\frac{1}{g}} \text {. }
$$


Now both these formulæ for $\sigma$ are derived by combining a theoretical equation with empirical formulæ, and in the theoretical equation molecular attraction is supposed not to exist. But the effect of molecular attraction on viscosity will partly account for the apparent shrinkage of molecules, because slowly moving molecules coming near to one another without actually colliding are swung round their collmon centre of mass by the action of molecular force, and are affected as regards their subsequent paths, as if they had suffered a collision. At high velocities two molecules, which pass close to one another without actual collision, shoot past one another with only a slight mutual deflexion; thus the molecules behave as if they had larger volumes at lower temperatures. This effect of molecular force on the temperature-variation of viscosity is a difficult problem in the kinetic theory of gases, which remains yet to be worked out. Steps towards its solution have been taken by Maxwell (Phil. Trans. 1867) and by L. Natanson (Kinetische Theorie unvollkommener Gase), but no definite enough result has yet been reached to give a quantitative estimate of the importance of molecular force in viscosity of gases. Yet from a general point of view we may feel pretty sure that in the case of hydrogen the large apparent shrinkage of the molecules cannot be traced entirely to the effect of molecular force, and must be regarded as a true phenomenon. If we take our deduction from Barus's experiments,

$$
\sigma_{273} / \sigma_{\theta}=(\theta / 273)^{\frac{1}{12}},
$$

we notice that, although $\theta$ ranges from $273^{\circ}$ to $1489^{\circ}$, yet $\theta^{\frac{1}{2}}$ ranges only from $1 \cdot 60$ to $1 \cdot 84$; and thus, while the formula for $\sigma$, if supposed to hold down to absolute zero, would give the impossible result of $\sigma$ being infinite at zero, this impossible result is merely the consequence of trying to extrapolate with an empirical formula over a range seven times as great as that covered by experiment.

Accordingly, we can get no definite assistance from the present state of the theory of viscosity of gases, but only a general assurance that molecules shrink with rising temperature. For the law of shrinkage of the molecules of solids I will take the expression $\mathbf{E}=\mathrm{E}_{0} /\left(1+b^{\prime} \theta\right)$, which, up to the melting-point, may more conveniently be used in the form $\mathbf{E}=\mathbf{E}_{0}\left(1-b^{\prime} \theta\right)$, so that $e-\mathbf{E}=\mathrm{E}_{0}\left(b+b^{\prime}\right) \theta$.

The steps in the differentiation for finding the bulk-modulus are not affected by the variability of $E$ with temperature: as before, we have (6), 


$$
\begin{aligned}
k & =\frac{2 e}{9}\left\{\frac{4 \mathrm{D}}{e^{3}(e-\mathrm{E})}-\frac{\mathrm{D}}{e^{2}(e-\mathrm{E})^{2}}\right\} \\
& =-\frac{2 \mathrm{D}}{9 \mathrm{E}_{0}^{3}}\left\{\frac{1}{\left(b+b^{\prime}\right)^{2} \theta^{2}}-\frac{4}{\left(b+b^{\prime}\right) \theta}\right\} \\
& =\frac{-2 \mathrm{~J}_{e m}}{9\left(b+b^{\prime}\right)^{2} \theta m / \rho}\left\{1-4\left(b+b^{\prime}\right) \theta\right\} ; . . .
\end{aligned}
$$

whence, using the mean of the quasi-experimental values of $k$ : given above as $k_{1}, k_{2}, k_{3}$, and the other known experimental terms, we can get the values of $b^{\prime}$ for the metals.

But our expression for the latent heat will be altered by the variability of $\mathrm{E}$. We bave to find at what rate the melting-pressure varies with the melting-temperature for a collection of molecules that shrink with rising temperature. Now melting has chiefly to do with the mobility of the individual molecules. Each molecule of a solid, if it is not close to the surface, moves as if it were free from the action of molecular force, because the attractions of the molecules all round it equilibrate one another. Let us conceive molecular force not to exist, that is conceive a perfect gas compressed till its molecules hem one another into fixed mean positions, this will be identical with our solid; except that molecular pressure is replaced by external pressure, the condition of the individual molecules is the same. Now melting occurs when the molecules escape from their domains, and suppose this happens for values $e, \mathbf{E}, p$, and $\theta$ of the variables : it is desired to find how much the temperature is to be raised to reach the melting-poirt when $p$ is increased to $p+d p$. It is obvious that $e / \mathbf{E}$ must remain constant, and the only other condition is that the collisional pressure increase must be equal to $d p$. Hence, if in our equation we ignore the molecular-force term and find $d p / d \theta$ on the supposition that $e / \mathrm{E}$ is constant, we can take it as $d p / d \theta$ in the thermodynamic relation $\lambda=\theta\left(v_{2}-v_{1}\right) d p / d \theta$,

$$
\theta d p / d \theta=\frac{2 \mathrm{D}}{3 e^{3}(1-\mathrm{E} / e)}\left(1-\frac{3 \theta}{e} \frac{d e}{d \theta}\right) .
$$

But $e / \mathrm{E}=\mu$ say, and $\mathrm{E}$ is a function of $\theta$ only, so that $d e / d \theta=\mu d \mathrm{E} / d \theta=-\mu \mathrm{E}_{0} b^{\prime}$,

$$
\therefore d e / e d \theta=-b^{\prime} \mathrm{E}_{0} / \mathbf{E}=-b^{\prime}\left(1+b^{\prime} \dot{\theta}\right) \text {. }
$$

Hence at the melting-point,

$$
\begin{aligned}
\lambda & =\frac{\left(v_{2}-v_{1}\right) 2 \mathrm{D}}{3 e^{2}(e-\mathrm{E})}\left\{1+3 b^{\prime} \mathrm{T}\left(1+b^{\prime} \mathrm{T}\right)\right\} \\
& =\frac{2 c m\left(v_{2}-v_{1}\right)\left\{1+3 b^{\prime \prime} \mathrm{T}\left(1+b^{\prime} \mathrm{T}\right)\right\}}{3\left(b+b^{\prime}\right) m^{\prime} / \rho} \text { calories. }
\end{aligned}
$$


If we now compare our expressions (11) and (12) for the bulk-modulus and latent heat with those which before gave results respectively 65 and 4.75 times too large, and neglect for a moment the small terms, we see that in a general way the theoretical bulk-modulus (11) and the experimental would agree if $b^{\prime}$ were put equal to $7 b$ as $8^{2}=64$, and the theoretical latent heat (12) would agree with the experimental if $b^{\prime}=3 \cdot 75 b$. But if in (11) and (12) we take account of the small terms and solve for $b^{\prime}$, we find that on the average the relation $b^{\prime}=6 b$ will bring the theoretical values of both the bulk-modulus and latent heat into accord with the experimental. The best way of showing this will be to tabulate the values calculated from (11) and (12) by means of the relation $b^{\prime}=6 b$, and compare them with the experimental. For the bulk-modulus the mean of $k_{1}, k_{2}, k_{3}$ given before is taken as the experimental value.

$$
10^{-6} \text { times the Bulk-modulus. }
$$

\begin{tabular}{|c|c|c|c|c|c|c|c|c|c|}
\hline Quasi-Exp... & $\begin{array}{c}\text { Cu. } \\
1550\end{array}$ & $\begin{array}{l}\mathrm{Ag} . \\
810\end{array}$ & $\begin{array}{r}A u . \\
1370\end{array}$ & $\begin{array}{l}\mathrm{Mg} . \\
325\end{array}$ & $\begin{array}{l}\mathrm{Zn} . \\
625\end{array}$ & $\begin{array}{l}\text { Al. } \\
565\end{array}$ & $\begin{array}{l}\mathrm{Pb} . \\
174\end{array}$ & $\begin{array}{c}\text { Fe. } \\
1660\end{array}$ & $\begin{array}{l}\text { Pt. } \\
790\end{array}$ \\
\hline heory ... & 1710 & 920 & 1790 & 310 & 410 & 560 & 216 & 3740 & 5310 \\
\hline atio .... & $1 \cdot 1$ & $1 \cdot 1$ & $1 \cdot 3$ & .96 & 66 & $1 \cdot 0$ & $1 \cdot 2$ & $2 \cdot 2$ & $6 \cdot 7$ \\
\hline
\end{tabular}

On the whole the values of the ratio show that $b^{\prime}=6 b$ brings experiment and theory into good accord as regards bulk-modulus.

\section{Latent Heat.}

\begin{tabular}{|c|c|c|c|c|c|}
\hline $\mathrm{Ex}$ & $\begin{array}{c}\mathrm{Ag} . \\
16 \cdot 2\end{array}$ & $\begin{array}{r}\mathrm{Zn} . \\
24.5\end{array}$ & $\begin{array}{c}\mathrm{Cd} . \\
12 \cdot 5\end{array}$ & $\begin{array}{c}\text { Hg. } \\
2 \cdot 8\end{array}$ & $\begin{array}{c}\mathrm{Sn} . \\
12 \cdot 8\end{array}$ \\
\hline heory... & 172 & $23 \cdot 7$ & $10 \cdot 4$ & 1.9 & $8 \cdot 0$ \\
\hline atio ... & $1 \cdot 06$ & .97 & $\cdot 83$ & $\cdot 67$ & $\cdot 63$ \\
\hline
\end{tabular}

If, for the reasons given before, we attach less weight to the values for mercury and tin, the value of the ratio for the other four metals is satisfactorily near to 1.

4. Further evidence of the Alteration of Molecules with Change of Temperature.-It is important now to see what further justification there is for the idea that molecules shrink with rising temperature. The small amount by which metals expand on being heated from absolute zero to their meltingpoints, namely about 2 per cent. of their linear dimensions, has made it difficult to form a mechanical conception of molecular behaviour during melting, because if the molecules are really at rest against one another at absolute zero it is hard 
to see how an increase of only 2 per cent. in the distance apart of the molecules can give them the freedom and mobility of the liquid state. But if while heat is driving the molecules apart from one another it is also shrinking each, the difficulty disappears, for if the shrinkage is six times the expansion then at the melting-point the distance apart of the molecules is nearly 16 per cent. greater than the diameter of the molecule; that is to say, the complete swing of the vibration is 16 per cent., and when two molecules have swung to their greatest distance apart that distance will be 16 per cent. greater than the diameter of the molecule. This is sufficient to make melting quite comprehensible. Let us take a definite instance : suppose four molecnles at absolute zero in contact so that their centres form a square of side $\mathrm{E}_{0}$, and as the temperature rises, suppose each to oscillate along a diagonal, then the maximum distance apart of the opposite molecules becomes $1 \cdot 16 \sqrt{2}$ times the diameter of the molecule, that is 1.63 times, so that a molecule vibrating at right angles to the plane of the square towards its centre, would, if it arrived at the right moment, find an open space whose narrowest part is 63 times its own diameter. This shows how, in the various combinations possible in a large number of molecules, several can occur favourable to the escape of a single molecule in a neighbourhood, and a sufficient number of such escajes, even though a small number, would upset the stability of the whole system, which falls into the mobility of a liquid. This theory of melting can be roughly tested by experiment; it is only necessary to agitate a number of equal spheres in a closed box and observe what ratio the free space bears to the volumes of the spheres when each sphere acquires a noticeable amount of mobility. I took a box which, when closed, just held 100 marbles, with five specially coloured ones placed near the centre, and then noted how many had to be removed from the box to allow the five coloured ones to seatter on agitation ; a slight motion of the five as a body was not accepted as a sign of mobility. It was necessary to remove 16 to get slow and partial scattering of the central 5 , and 20 or 25 to get quick and decided scattering. Thus for mobility among a set of marbles the free volume must be between 25 and 33 per cent. of the volume of the marbles. Of course the circumstances are in most ways vastly different from those of perfectly rebounding swiftly moving molecules, but the comparison was worth making in passing. In the case of molecules mobility is reached when the free volume is $1 \cdot 16^{3}-1$, or 50 per cent. of the volume of the molecules. But the melting volumes of the metals give more definite evidence. 
According to the kinetic theory of solids now under discussion, it is necessary, if the molecules of different metals are not much different in shape, that the ratio of the domain of the molecule at the melting-point to the volume of the molecule should be the same for all, because melting is merely the result of the domain reaching a size at which the molecule escapes from it. Hence $(1+b \mathrm{~T})\left(1+b^{\prime \prime} \mathrm{T}\right)$ is to be the same for all, that is $b \mathrm{~T}+b^{\prime} \mathrm{T}$ is to be constant; but $b^{\prime}=6 b$, hence $b \mathrm{~T}$ is to be constant. Now in a previous paper (Phil. Mag. Oct. 1890) I mentioned that $b \mathrm{~T} \mathrm{~m}^{\frac{1}{\xi}}$ is nearly constant for the metals, and used the result to calculate the periods of vibration of the molecules of solids at their melting-points with satisfactory results. But the range in the value of $m^{\frac{7}{6}}$ for the metals is not great, and it will be worth while tabulating side by side the values of $b \mathrm{~T}$ and $b \mathrm{~T} m$. Fizeau's values of $b$ are used.

\begin{tabular}{|c|c|c|c|c|c|c|}
\hline & Cu. & Ag: & $\mathrm{Au}$. & $\mathrm{Mg}$. & $\mathrm{Zn}$. & Cd. \\
\hline $1000 \mathrm{bT}$. & 22 & 24 & 19 & 27 & 20 & 18 \\
\hline $1000 b \mathrm{~T} m^{\frac{7}{6}} \ldots$ & 45 & 52 & 45 & 47 & 40 & 40 \\
\hline & In. & $\mathrm{Tl}$. & Sn. & $\mathrm{Pb}$. & Fe. & Co. \\
\hline $000 \mathrm{bT} \ldots . .$. & 19 & 17 & 11 & 18 & 25 & 26 \\
\hline $1000 b \mathrm{~T} m^{\frac{1}{5}} \ldots$ & 41 & 41 & 25 & 43 & 49 & 51 \\
\hline
\end{tabular}

\begin{tabular}{|c|c|c|c|c|c|}
\hline & Ru. & $\mathrm{Rh}$. & $\mathrm{Pd}$. & Os. & Ir. \\
\hline $1000 b \mathrm{~T} \ldots . . .$. & 20 & 19 & 21 & 18 & 16 \\
\hline $000 b \mathrm{~T}^{\frac{1}{6}} \ldots$ & 43 & 42 & 45 & 44 & 37 \\
\hline
\end{tabular}

This comparison shows that the requirement of theory that $b \mathrm{~T}$ should be constant is approximately satisfied, $b \mathrm{~T}$ having a mean value $\cdot 021$ when tin is excluded; but as $b \mathrm{~T} m^{\frac{1}{6}}$ is more nearly constant, having a mean value $\cdot 044$, it is evident that the power of a molecule to break away from its domain depends slightly on its mass, that dependence being expressed in the empirical relation $b \mathrm{~T} m^{\frac{7}{5}}=$ constant.

5. Periods of Vibration of the Molecules of Metals.-As the vibrational motion of the molecules is fundamental to the kinetic theory of solids here unfolded, it will be well at this stage to secure such support for the theory as is given by the harmonic relations I have shown to exist among the periods of vibration of the molecules of metals at their melting-points (Phil. Mag. Oct. 1890). The full swing of a molecule in one direction is

$$
e-\mathrm{E}=\mathrm{E}_{0}\left(b \mathrm{~T}+b^{\prime} \mathrm{T}\right)=7 \mathrm{E}_{0} b \mathrm{~T} .
$$


Let $\mathbf{M}$ stand for the molecular weight of a molecule referred in the ordinary way to that of hydrogen, and let $\mathrm{H}$ denote the real mass of a molecule of hydrogen, then the swing of a molecule is.

$$
7(\mathrm{MH} / \rho)^{\frac{1}{3}} \cdot 044 / \mathrm{M}^{\frac{1}{5}} \text {. }
$$

But its translatory kinetic energy is

$$
\begin{aligned}
& \frac{1}{2} \mathrm{MH} v^{2}=\mathrm{J}_{c} \mathrm{MHT}, \\
\therefore \quad & v=\sqrt{2} \mathrm{~J}_{c} \mathrm{MT} / \mathrm{M},
\end{aligned}
$$

and accordingly the period of a complete vibration is

$$
p=\frac{\mathrm{M}^{\frac{3}{3}}}{\rho^{\frac{1}{3}} \mathrm{~T}^{\frac{1}{2}}} \cdot \frac{2 \times 7 \times 044 \mathrm{H}^{\frac{1}{3}}}{\sqrt{2} \mathrm{JcM}} .
$$

For comparative values we can drop the whole numerical factor and take $\mathrm{M}^{\frac{2}{3}} / \rho^{\frac{1}{3}} \mathrm{~T}^{\frac{1}{3}}$ as measuring the relative periods. In the subjoined table I replace the erroneously copied values for the beryllium family, given in my former paper, by the correct ones.

$$
\text { Relative Periods, } \mathrm{M}^{\frac{2}{3}} / \rho^{\frac{1}{3} \mathrm{~T}_{\frac{1}{2}}} \text {. }
$$

$\begin{array}{ccccccccc}\text { Li. } & \text { Na. } & \text { K. } & \text { Rb. } & \text { Cs. } & \text { Cu. } & \text { Ag. } & \text { Au. } & \\ \cdot 205 & \cdot 43 & \cdot 66 & \cdot 96 & 1 \cdot 22 & \cdot 21 & \cdot 29 & \cdot 35 & \\ \text { Be. } & \text { Mg. } & \text { Ca. } & \text { Sr. } & \text { Ba. } & \text { Zn. } & \text { Cd. } & \text { Hg. } & \\ \cdot 107 & \cdot 22 & \cdot 34 & \cdot 51 & \cdot 63 & \cdot 32 & \cdot 47 & \cdot 94 & \\ . \text { Al. } & \text { Ia. } & \text { Ga. } & \text { In. } & \text { Tl. } & & & & \\ \cdot 20 & \cdot 55 & \cdot 54 & \cdot 57 & \cdot 65 & & & & \\ \text { F.. } & \text { Co. } & \text { Ni. } & \text { Ru. } & \text { Rh. } & \text { Pd. } & \text { Os. } & \text { Ir. } & \text { Pt. } \\ \cdot 16 & \cdot 16 & \cdot 17 & \cdot 21 & \cdot 20 & \cdot 24 & \cdot 23 & \cdot 25 & \cdot 27\end{array}$

In the $\mathrm{Li}$ family the periods run as $1,2,3,4.5,6$ with the copper sub-family connected; in the Be family the periods run as $1,2,3,4 \cdot 5$, 6 , with the zinc sub-family related in somewhat the same way as the copper to the lithium, but not exactly. $\mathrm{Al}$ and $\mathrm{La}$ have periods nearly as 2 to 6 . The other periods do not call for comment at present; but it is worth noting that the periods of the Be family are half those of the : Li family. These beautiful harmonic relations almost amount to a proof that the expansion of the metals is a true measure of the amplitude of vibration of their molecules. It will be interesting to try to get an absolute value of one of these periods, and compare it with known periods of light- and heat-vibrations. Sir W. Thomson estimates that 
there are between $3 \times 10^{24}$ and $10^{26}$ molecules in a cubic centimetre of ordinary liquids and solids. Now the limiting volume of a gramme of hydrogen is about 4 cubic centimetres, so that if we take $10^{25}$ as the number of molecules in a cubic centimetre of hydrogen $\mathrm{H}=2.5 \times 10^{-26}$ gramme, and the numerical factor dropped above becomes $8 \times 10^{-14}$, which makes the absolute period of the Lithium molecule $1 \cdot 6 \times 10^{-14}$ second; that is to say, there are $6 \times 10^{13}$ complete vibrations of the molecule in a second. Now the A line of the spectrum represents $3.945 \times 10^{14}$ vibrations, and the $\mathrm{H}_{2}$ line $7 \cdot 628 \times 10^{14}$ vibrations per second, while in the dark part of the spectrum Langley has found a line of $1 \cdot 1 \times 10^{14}$, and in the radiation of bodies below $100^{\circ} \mathrm{C}$. a line of $2 \times 10^{13}$ vibrations in a second. Accordingly, the periods of vibration of the molecules of the metals at their melting-points fall within the limits of actually measured periods of non-luminous vibrations. This fact supplies good general verification to the theory, and shows how interesting a bolometric study of the radiation of solids just about to melt would be.

6. Comparison of the Theoretical Variation of Young's Modulus with Temperature with the Experimental.-The next subject to apply the theory to with advantage is the theoretical law of variation of Young's modulus with temperature for comparison with the experimental results given in the introduction. The metals, as will yet be shown, may be assumed to be approximately isotropic. In isotropic solids the relation between $q, n$, and $k$ is $q=9 k n /(3 k+n)$. Now in the introduction it was shown for all the metals that $n / \mathrm{N}=1-(\theta / \mathrm{T})^{2}$, where $\mathrm{N}$ is rigidity at absolute zero, and from the theoretic equation (11) we have the values of $k$ at all temperatures, and

$$
q=\frac{3 \mathrm{~N}\left\{1-(\theta / \mathrm{T})^{2}\right\}}{1+\mathrm{N}\left\{1-(\theta / \mathrm{I})^{2}\right\} / 3 k} .
$$

Now, according to (11), $k$ is infinite at absolute zero, which we will interpret to mean that it is very large, and hence $Q$ the value of Young's modulus $q$ at absolute zero is $3 \mathrm{~N}$. Accordingly we have

$$
\mathrm{Q}\left\{1-(\theta / \mathrm{T})^{2}\right\}(1 / q-1 / 9 k)=1 \quad . \quad .
$$

as the definitely prescribed law of variation of Young's modulus with temperature. Because of this definite theoretic relation I did not, in the introduction, dwell on the demonstration of any empiric relation, but the values given for different temperatures and different metals will now furnish a 
good test of the kinetic theory of solids. The best way to apply the test will be to calculate for each metal from the observed values of $q$ and the theoretic values of $k$ the values of $Q$,- these should come the same. For example, according to my experiments on zinc the values of $q 10^{-6}$ at absolute temperatures $289^{\circ}, 333^{\circ}$, and $376^{\circ}$ are 821,758 , and 682 , and the corresponding values of $Q 10^{-6}$ are 1280,1320 , and 1320 , which satisfy well the requirement of constancy. Kiewiet's values for zinc, found by the method of bending at $283^{\circ}, 325^{\circ}$, and $353^{\circ}$, are 1031,975 , and 924 , which give for $\mathrm{Q} 10^{-6}$ the values 1710,1800 , and 1870 , which do not satisfy the condition of constancy. It will be seen from equation (13) that a good deal depends on a correct absolute value of $\%$, as well as on the relative values at different temperatures. For the other metals I will simply give the values of $\mathrm{Q} 10^{-6}$ calculated from 3 values of $q$ at temperatures from $289^{\circ}$ to $376^{\circ}$ on the absolute scale. For tin 880,890 , and 990 , and according to Kiewiet 740,750 , and 720 ; lead 326,332 , and 339 ; magnesium 439,443 , and 433 ; copper 1590 and 1540 ; aluminium 818 and 839 ; alumininm mannealed 735,746 , and 752 ; and iron 2253,2241 , and 2226 . It is evident that experimental uncertainties do not at present let us expect any better agreement between theory and experiment than that just shown. But there is another form in which the same comparison can be made. One of the results of equation (13) is that Young's modulus at absolute zero is 3 times the rigidity. Now by means of (13) we can from the mean values of $q$ at $15^{\circ} \mathrm{C}$. given in Table VII. calculate the values of $Q$, and compare them with the values of $N$, given in Table V.

TABLE VIII.

$10^{-6} \mathrm{Q}$ or $10^{-6}$ times Young's modulus at absolute Zero.

$\begin{array}{lccccccc} & \text { Cu. } & \text { Ag. } & \text { Au. } & \text { Mg. } & \text { Zn. } & \text { Cd. } & \text { Al. } \\ \text { Q..... } & 1390 & 860 & 840 & 490 & 1490 & 1040 & 840 \\ \text { Q/N... } & 3 \cdot 1 & 2 \cdot 9 & 2 \cdot 9 & 3 \cdot 0 & 3 \cdot \tilde{3} & & 3 \cdot 2 \\ & & & & & & & \\ \text { S..... } & 700 & \text { Pb. } & \text { Fe. } & \text { Ni. } & \text { Co. } & \text { Pd. } & \text { Pt. } \\ \text { Q/N... } & 3.5 & 2 \cdot 3 & 2170 & 2460 & 1970 & 1130 & 1690 \\ & & 2 \cdot 8 & 3 \cdot 2 & & & 2 \cdot 6\end{array}$

With the exception of platinum, the only metals for which the departure of $\mathrm{N} / \mathrm{Q}$ from the value 3 is beyond the limits of experimental error are zinc, tin, and lead, the metals for which the results of different experimenters disagree most, as 
shown in the compilation of data given in the introduction. These disagreements would seem to indicate that even if these metals can be got in an approximately isotropic state at all, their isotropy is easily disturbed, but this point will be returned to immediately. The mean value for the ratio $Q / N$ for the 7 metals $\mathrm{Cu}, \mathrm{Ag}, \mathrm{Au}, \mathrm{Mg}, \mathrm{Al}, \mathrm{Fe}$, and $\mathrm{Ni}$ is $3 \cdot 0$, and accordingly it appears that these metals may be regarded as isotropic, and as having molecules so nearly incompressible as to give the relation $Q=3 \mathrm{~N}$ characteristic of incompressible molecules.

The study of the ratio $\mathrm{Q} / \mathrm{N}$ leads naturally to the famous controversy amongst elasticians as to whether there is a fixed value of the ratio of lateral contraction to longitudinal extension for all isotropic bodies subject to traction; but this matter had better be postponed a little until we have seen what account the kinetic theory can give of rigidity. This is the most important point in a theory of solids, and as I have already pointed out that no static theory can give an adequate explanation of rigidity, there is great interest in seeing how the kinetic theory will fare.

7. Rigidity according to the Kinetic Theory.-The fundamental equation for a solid, homogeneous and isotropic and free from external force is

or

$$
\text { (4) } \frac{2 \mathrm{D}}{3 e^{2}(e-\mathrm{E})}-\frac{1}{6 e^{3}} \Sigma r \phi(r)=0 \text {, }
$$

$$
\frac{2 \mathrm{D}}{3 e^{2}(e-\mathrm{E})}-\mathrm{B} \rho^{2}=0 \text {. }
$$

Suppose the solid now subjected to a pure: shearing stress specified by a traction $\mathrm{P}$ parallel to the axis of $x$, and a pressure $\mathrm{P}$ parallel to that of $y:$ the traction counts as a pressure $-\mathrm{P}$, and the corresponding strains are an elongation of $e$ to $\xi$ parallel to $x$, and a contraction of $e$ to $\eta$ parallel to $y$. The strained body is no longer isotropic, and equations (3) are applicable to it if we write $-\mathrm{P}$ for $\mathrm{P}$, and put $\mathrm{Q}=\mathrm{P}$ and $\mathrm{R}=0$. Let $\xi=e+\delta \xi$, and $\eta=e+\delta \eta$, and $\xi=e$, then $\delta \eta=-\delta \xi$. The effective kinetic energies in different directions are no longer equal each to $\mathrm{D}$, but become $\frac{1}{2} m n u^{2}, \frac{1}{2} m v^{2}$, and $\frac{1}{2} m w^{2}$, which will be denoted by $\mathrm{D}_{1}, \mathrm{D}_{2}$, and $\mathrm{D}_{3}$, and let $\mathrm{D}_{1}=\mathrm{D}+\delta \mathrm{D}_{1}$ and so on. We do not know at present how we are to express the condition that the shear is made at constant temperature, for when the effective kinetic energy is different in different directions, what is the relation between these different energies and temperature? All that we can assert is $\delta D_{2}=-\delta D_{1}$. Now the shear produces a change of volume 
only of the second order, so that $\Sigma r \phi(r) / 6 e^{3}$ may be regarded as unaltered, and after the shear we have

$$
\frac{2}{3} \cdot \frac{\mathrm{D}+\delta \mathrm{D}_{1}}{(e+\delta \eta) e(e+\delta \xi-\mathrm{E})}+\mathrm{P}-\frac{1}{6 e^{3}} \Sigma r \phi(v)=0 .
$$

Replace the last term by its value $2 \mathrm{D} / 3 e^{2}(e-\mathrm{E})$, and expand the first term, then

$$
\begin{aligned}
-\mathrm{P} & =\frac{2}{3} \frac{\mathrm{D}}{e^{2}(e-\mathrm{E})}\left(\frac{\delta \mathrm{D}_{1}}{\mathrm{D}}-\frac{\delta \eta}{e}-\frac{\delta \xi}{e-\mathrm{E}}\right), \\
& =\frac{2}{3} \frac{\mathrm{D}}{e^{2}(e-\mathrm{E})}\left(\frac{\delta \mathrm{D}_{1}}{\mathrm{D}}+\frac{\delta \xi}{e}-\frac{\delta \xi}{e-\mathrm{E}}\right) .
\end{aligned}
$$

$\mathrm{P}$ is the shearing stress, and $2 \delta \xi / e$ is the shear, so that the rigidity $n$ is $\mathrm{Pe} / 2 \delta \xi$,

$$
n=-\frac{1}{3} \frac{\mathrm{D}}{e^{2}(e-\mathrm{E})}\left(\frac{\delta \mathrm{D}_{1}}{\delta \xi} \frac{e}{\mathrm{D}}-\frac{e}{e-\mathrm{E}}+1\right) .
$$

This equation gives a statement of what rigidity is according to the kinetic theory; it depends on the rate of change of the effective kinetic energy in a direction with the change of the distance apart of the molecules in that direction. This ratio of $\delta \mathrm{D}_{1}$ to $\delta \xi$ is fundamental in the theory of solids; the condition for zero rigidity or fluidity is

$$
e \delta \mathrm{D}_{1} / \mathrm{D} \delta \xi=e /(e-\mathrm{E})-1 \text {. }
$$

It would be possible to calculate the ratio of $\delta D_{1}$ to $\delta \xi$ on purely theoretical ground with the aid of suppositions as to the distribution of kinetic energy in different directions and its relation to temperature, but this is not worth doing at present; it will only be shown shortly that even in solids $\delta \mathrm{D}_{1} / \delta \xi$ differs only from the large number $e /(e-\mathrm{E})$ by a small number, that is, a number not much greater than 1. But first it may be as well to determine Young's modulus from our equations in the same way as we have just found the rigidity. It is only necessary to put $-\mathrm{P}$ for $\mathrm{P}_{1}, \mathrm{Q}=0$, $\mathrm{Z}=0, \zeta=e+\delta \zeta, \delta \zeta=\delta \eta=-\sigma \delta \xi$, and replace $\Sigma r \phi(r) / 6 e^{3}$ by $\mathrm{B} m^{2} / \xi^{2} \eta^{2} \zeta^{2}$, and to expand, to get the following equations in which the variables are accented to distinguish the conditions of variation from the former ones :

$$
\begin{aligned}
& -\mathrm{P}=\frac{2}{3} \frac{\mathrm{D}}{e^{2}(e-\mathrm{E})}\left(\frac{\delta \mathrm{D}_{1}^{\prime}}{\mathrm{D}}+\frac{\delta \eta^{\prime}+\delta \xi^{\prime}+2 \delta \xi^{\prime}}{e}-\frac{\delta \xi}{e-\mathrm{E}}\right), \\
& \left.q=\frac{\mathrm{P} e}{\delta \xi^{\prime}}=-\frac{2}{3} \frac{\mathrm{D}}{e^{2}(e-\mathrm{E})}\left(\frac{\delta \mathrm{D}_{1^{\prime}} e}{\delta \xi^{\prime} \mathrm{D}}-2 \sigma+2-\frac{e}{e-\mathrm{E}}\right)\right\} \\
& \frac{\delta \mathrm{D}_{2}^{\prime}}{\mathrm{D}}=\frac{\delta \mathrm{D}_{3}^{\prime}}{\overline{\mathrm{D}}}=\left(3 \sigma-1-\frac{\sigma e}{e-\mathrm{E}}\right)^{\frac{\delta \xi}{e}} \text {. }
\end{aligned}
$$


Now at absolute zero our collection of molecules is supposed to be practically incompressible, so that $Q=3 \mathrm{~N}$, and $\sigma=\frac{1}{2}$; hence for $\mathrm{N}$ we have the two values, the limit of

and the limit of

$$
\frac{1}{3} \frac{\mathrm{D}}{e^{2}(e-\mathrm{E})}\left(\frac{\delta \mathrm{D}_{1}}{\delta \xi} \frac{e}{\mathrm{D}}-\frac{e}{e-\mathrm{E}}+1\right)
$$

$$
\frac{2}{9} \frac{\mathrm{D}}{e^{2}(e-\mathrm{E})}\left(\frac{\delta \mathrm{D}_{1}^{\prime \prime}}{\delta \xi^{\prime}} \frac{e}{\mathrm{D}}-\frac{e}{e-\overline{\mathrm{E}}}+1\right) .
$$

These two forms and the value of $\delta \mathrm{D}_{2}^{\prime}$ above, which at absolute zero is given by $2 e \delta \mathrm{D}_{2}^{\prime} / \mathrm{D} \delta \xi^{\prime}=1-e /(e-\mathrm{E})$, suggest that both $e \delta \mathrm{D}_{1} / \delta \xi \mathrm{D}$ and $e \delta \mathrm{D}_{1}^{\prime} / \delta \xi^{\prime} \mathrm{D}$ are nearly equal to $e /(e-\mathrm{E})$. If in the case of Young's modulus the condition for constant temperature were that the mean of the effective kinetio energies in all directions is constant or

$$
\delta\left(\mathrm{D}_{1}{ }^{\prime}+\mathrm{D}_{2}{ }^{\prime}+\mathrm{D}_{3}^{\prime}\right)=0
$$

then, from the values of $\delta \mathrm{D}_{2}{ }^{\prime}$ and $\delta \mathrm{D}_{3}{ }^{\prime}$ we get

$$
\frac{e \delta \mathrm{D}_{1}^{\prime}}{\delta \xi^{\prime} \mathrm{D}}=\left(\frac{2 \sigma e}{e-\mathrm{E}}+2-6 \sigma\right)
$$

which at absolute zero would make $q$ vanish, and therefore cannot be quite correct, but proves at all events that at absolute zero $e \delta \mathrm{D}_{1}^{\prime} / \mathrm{D} \delta \xi^{\prime}$ is nearly equal to $e /(e-\mathrm{E})$. The two expressions for $\mathrm{N}$ show that at absolute zero we may put

$$
\frac{e \delta \mathrm{D}_{1}^{\prime}}{\mathrm{D} \delta \delta_{\xi^{\prime}}^{\prime \prime}}=\frac{e}{e-\mathrm{E}}+\mathrm{A},
$$

where $\mathrm{A}$ is a constant the same for all bodies, and

$$
\frac{e \delta \mathrm{D}_{1}}{\mathrm{D} \delta \xi}=\frac{e}{e-\mathrm{E}}+\mathrm{B},
$$

where $B$ is the same for all bodies, and

$$
\mathrm{B}+1=\frac{2}{3}(\mathrm{~A}+1) \text {; }
$$

$A$ and Bare both numbers not large compared to 1 . Then $\mathrm{N}$ is the limiting value of

$$
\frac{1}{3} \frac{\mathrm{D}(\mathrm{B}+1)}{e^{2}(e-\mathrm{E})}
$$

which, with $\mathrm{D}=\mathrm{J} \operatorname{con} \theta$ and $e-\mathrm{E}=7 b \theta \mathrm{E}_{0}$, gives

$$
\mathrm{N}=\frac{\mathrm{J} c m(\mathrm{~B}+1)}{216 m / \rho} ; . . . \quad .
$$


em is constant, so that we have this result, that the product $\mathrm{Nbm} / \rho$ is the same for all metals, and so also the product Qbm/p. The best manner of testing this last theoretical conclusion will be to tabulate the values of $7 \mathrm{Q} b \mathrm{~m} / \mathrm{J} \mathrm{cm} \rho$ and $21 \mathrm{~N} b m / J c m \rho$, and see whether all the metals give the same value for $B+1$.

\begin{tabular}{|c|c|c|c|c|c|}
\hline $\mathrm{Qbm} / \mathrm{J} c m \rho$ or $\mathrm{B}+1 \ldots 4^{\circ}{ }^{\mathrm{Cu}}$ & $\begin{array}{l}\text { Ag. } \\
4 \cdot 6\end{array}$ & $\begin{array}{l}\text { Au. } \\
3 \cdot 2\end{array}$ & $\begin{array}{l}\mathrm{Mg} . \\
5 \cdot 0\end{array}$ & $\begin{array}{c}\mathrm{Zn} . \\
10 \cdot 5\end{array}$ & $\begin{array}{c}\mathrm{Od} . \\
10 \cdot 9\end{array}$ \\
\hline $\mathrm{N} b m / \mathrm{J} c m \rho$ or $\mathrm{B}+1 \ldots 4 \cdot 5$ & $4 \cdot 7$ & $3 \cdot 2$ & $5 \cdot 0$ & $9 \cdot 0$ & \\
\hline$b m / J_{c m p}$ or $\mathrm{B}+1 \ldots 6^{\text {Sn. }}$ & $\begin{array}{l}\mathrm{Pb} . \\
3 \cdot 7\end{array}$ & $\begin{array}{l}\mathrm{Fe} \\
4 \cdot 8\end{array}$ & $\begin{array}{l}\mathrm{Ni} \\
5 \cdot 4\end{array}$ & $\begin{array}{l}\text { Co. } \\
4 \cdot 4\end{array}$ & $\begin{array}{l}\mathrm{Pd} . \\
3 \cdot 2\end{array}$ \\
\hline $\mathrm{N} b m / \mathrm{J} e m \rho$ or $\mathrm{B}+15.4$ & $4 \cdot 7$ & $5 \cdot 1$ & $5 \cdot 2$ & & \\
\hline
\end{tabular}

The values for zine and cadmium are about double those for the other elements; if for the moment we take half of them as the true values, and take the mean of all, we get for $\mathrm{B}+1$ the value $4 \cdot 6$, the serious departures from which are in the case of gold $3 \cdot 2$, aluminium mean $5 \cdot 7$, tin mean $5 \cdot 8$, and palladium $3 \cdot 2$; in the case of palladium the fault lies probably in the absolute value of $g$ at $15^{\circ} \mathrm{C}$., which is the mean of Wertheim's static and kinetic values $980 \times 10^{6}$ and $1130 \times 10^{6}$, while before annealing they are $1180 \times 10^{6}$ and $1240 \times 10^{6}$. In the case of tin uncertain isotropy and experimental uncertainty explain the discrepance, but for gold and aluminium no adequate explanation is available except one suggested below. On the whole the theoretical conclusion is well borne out. In the case of zine and cadmium, the fact that the product is double the normal value is connected with the fact that the molecules of zinc and cadmium are known in the vaporous state to be monatomic. The product $7 \mathrm{Qbm} / \mathrm{J} \mathrm{cm} \rho$ is independent of $m$ the molecular mass; but as we do not know the dynamical significance of the constant $B+1$, we cannot tell whether or not it ought to be twice as large for monatomic molecules as for diatomic. The assertion that $\mathrm{B}+1$ must be the same for all metals goes on the assumption that they are dynamically similar systems of molecules at absolute zero; if they are not so, then the assertion no longer holds, and this may be the cause of the difference in the case of gold and aluminium.

The theoretical relation that $\mathrm{Q} b m / \rho$ is to be the same for all metals corresponds to Wertheim's empirical discovery that, if $q$ is Young's modulus at $15^{\circ} \mathrm{C} ., q(\mathrm{~m} / \rho)^{\frac{7}{3}}$ is approximately the same for all the metals (Ann. de Ch. et de Ph. sér 3. t. xii.). 202 
Tomlinson (Phil. Trans. 1883) has investigated the same relation. We have seen, in the experimental introduction, that the elastic properties of the metals correspond only at temperatures which are the same fraction of the melting-temperatures, and accordingly any relation connecting Young's modulus at a fixed temperature with molecular volume or domain must be empirical. Now as the coefficient of expansion $b$ is roughly proportional to the molecular domain $m / \rho$, the relation $\mathrm{Q} b m / \rho=$ constant becomes $\mathrm{Q}(m / \rho)^{2}=$ constant approximately, whence we see the origin of Wertheim's relation. There are various other approximate empirical relations discovered by different physicists which correspond to combinations of those already established on theoretical grounds in this paper, but there is no use in discussing them further.

8. Ratio of Lateral Contraction to Elongation in Young's Experiment.-We can now take up the famous question as to whether the ratio of lateral contraction to longitudinal extension in the Young's modulus experiment has the same value for all isotropic bodies, as asserted by various builders of statical molecular theories of elasticity, and as denied by the upholders of a science of elasticity apart from molecular considerations. As no statical theory founded on central forces can give a true account of rigidity, it seems useless to discuss a deduction of such theories, but the important point of getting a test for isotropy is involved. We see at once, from the results of the experimental introduction, that temperature is an important condition which has never been taken into account by the upholders of a constant ratio. In isotropic solids we have the following equations for $\sigma$ the ratio of contraction to elongation :-

$$
\sigma=(3 k-2 n) / 2(3 k+n) \text { and } \sigma=q / 2 n-1 .
$$

Now we have seen experimentally that

$$
n=\mathrm{N}\left\{1-(\theta / \mathrm{T})^{2}\right\},
$$

and by theory (11)

$$
k=-\frac{2}{9} \frac{\mathrm{J} c m(1-28 b \theta)}{49 b^{2} \theta m / \rho},
$$

so that $\sigma$ is a not simple function of the temperature. At absolute zero $k$ is according to our theory very large, and may be regarded as infinite, while $n$ remains finite, and therefore the value of $\sigma$ at absolute zero is $\frac{1}{2}$. But again at the melting-point $n$ is zero, while $k$ is finite, so that at the melting-point $\sigma$ again attains the value $\frac{1}{2}$. Now $\sigma$ has been 
measured directly for a few metals by Mallock (Proc. Roy. Soc. xxix.) by one method, and by Ämagat (Compt. Rend. cviii.) by a quite distinct method, and also by an indirect method. We will compare the values given by the first equation for $\sigma$ at $15^{\circ} \mathrm{C}$. with these experimental values.

\begin{tabular}{|c|c|c|c|}
\hline \multicolumn{4}{|c|}{ Values of $\sigma$ at $15^{\circ} \mathrm{C}$. } \\
\hline & $\mathrm{Cu}$. & $\mathrm{Zn}$. & \\
\hline Theory & $\cdot 38$ & $\cdot 17$ & \\
\hline Mallock & $\cdot 35$ & $\cdot 18$ & $\cdot$ \\
\hline Amagat & $\cdot 32$ & ... & \\
\hline
\end{tabular}

For iron theory gives a value $\cdot 41$, while Mallock found for steel the value $\cdot 25$, and Amagat for steel $\cdot 27$, but Okatow (Pogg. Ann. cxix.) has shown that steel according to its treatment gives values of $\sigma$ ranging from 275 to $\cdot 40$. If we calculate $\sigma$ according to the second equation above, using the mean experimental values of $q$ and $n$ at $15^{\circ}$, as given in Tables III. and VII. of the introduction, we get the following values:- $\mathrm{Cu} \cdot 42, \mathrm{Zn} \cdot 33, \mathrm{~Pb} \cdot 13, \mathrm{Fe} \cdot 33$. These values for zinc and lead are in complete disaccord with the experimental values, that for zinc being much too large, and that for lead much too small. Now in studying the values of the ratio $\mathrm{Q} / \mathrm{N}$, which ought theoretically to be 3 , we found for zinc $3 \cdot 5$, and for lead $2 \cdot 3$; but as the valtes of $Q$ and $N$ are both calculated from the mean values of $q$ and $n$ at $15^{\circ} \mathrm{C}$., it is very likely that there is experimental error in $q$ and $n$ as individual experimenters differ greatly in their values. Hence if 3 is the true value for $Q / N$ for all the metals, $q / n$ is wrong in the same proportion as $Q / N$; and if we multiply our values of $q / n$ by 3 , and divide by $Q / N$, and use the result in our second equation for $\sigma$, we shall see whether theory will thus eliminate the discrepancy above for zinc and lead as due to experimental uncertainty in $q / n$. Doing this, we get for $\mathrm{Ca} \cdot 38, \mathrm{Zn} 14, \cdot \mathrm{Pb} \cdot 47$, and $\mathrm{Fe} 42$, which agree well with the values given by the first equation for $\sigma$, and with the directly observed values of Mallock and Amagat.

This agreement of theory and experiment shows that all idea of a constant value for $\sigma$ in the case of isotropic bodies must be abandoned, and, what is much more important, that the metals are at least approximately isotropic. Amagat has already shown by some fine experimental work (Compt. Rend. cviii.) that some metals and alloys are isotropic, although his method of calculation makes the proof a little more perfect than it actually is. His method is to measure Young's modulus directly by statical experiments ; let $\mathrm{P}$ be the pressure 
applied longitudinally to a cylinder of length $\mathrm{L}$ producing a change of length $d \mathrm{~L}$, then

$$
q=\mathrm{PL} / d \mathrm{~L} \quad(a) .
$$

$\mathrm{He}$ also observed directly the change of volume of the cylinder in this experiment, but

$$
d \mathrm{~V}=(1-2 \sigma) \mathrm{VP} / q \quad(b) ;
$$

hence these two experiments give a direct determination of $\sigma$. Again, he compressed the hollow cylinder of radii $R$ and $R_{0}$ externally with hydrostatic pressure, and according to elastic theory, if the material of the cylinder is isotropic the change of volume is given by

$$
d \mathrm{~V}=(5-4 \sigma) \mathrm{R}^{2} \mathrm{PV} /\left(\mathrm{R}^{2}-\mathrm{R}_{0}{ }^{2}\right) q \quad(c) .
$$

Amagat used this equation and (b) to calculate $\sigma$ and $q$ from his data ; but a somewhat more trustworthy method would be to substitute the directly observed value of $q$ in $(c)$ and so obtain $\sigma$ for comparison with the direct value given by $(a)$ and $(b)$. To complete the available evidence for the isotropy of the metals and alloys I will reproduce Amagat's directly found values of $\sigma$ and $\sigma_{1}$, his values got from $(b)$ and $(c)$ as $\sigma_{2}$, and values which I have calculated from $(a)$ and $(c)$ as $\sigma_{3}$.

$$
\begin{array}{ccccccc} 
& & \text { Steel. } & \text { Copper. } & \text { Brass. } & \text { Delta. } & \text { Lead. } \\
\sigma_{1} & \ldots \ldots & \cdot 268 & \cdot 325 & \cdot 324 & \cdot 347 & \cdot 431 \\
\sigma_{2} & \ldots \ldots & \cdot 269 & \cdot 329 & \cdot 330 & \cdot 333 & \cdot 425 \\
\sigma_{3} & \ldots \ldots & \cdot 263 & \cdot 303 & \cdot 301 & \cdot 388 & \cdot 493
\end{array}
$$

The agreement is sufficient to justify the assumption of Isotropy on which equation $(c)$ is founded.

9. Dulong and Petit's Law, Joule and Kopp's Law, and the Equation for Conıpound Solids.-The physical meaning of Dulong and Petit's law of the constancy of the atomic heats of the elements has been a subject of speculation with chemists and physicists ever since its discovery. As the kinetic theory of gases in regarding temperature as proportional to kinetic energy of the molecule, required that apart from internal motion and work the molecular heats of all gases should be the same, it has been felt that a similar connexion between temperature and molecular kinetic energy is at the foundation of Dulong and Petit's law. The kinetic theory of solids makes this quite plain, that Dulong and Petit's law is simply the expression of the fact that temperature is proportional to the kinetic energy of the molecule, or, to be more accurate, of the atom. 'The meaning of Dulong and Petit's law becomes 
still clearer when taken in conjunction with that of Joule and Kopp, that the molecular heat of a solid compound is the sum of the atomic heats of the component atoms. This means that the freedom of the atom within the molecule is such that each atom vibrates independently of the others. On this basis it is easy to sketch the investigation of the equations for a solid compound. Let us consider a compound whose molecule consists of $n_{1}$ atoms of an element $A_{1}, n_{2}$ of $A_{2}$, and so on. Suppose a cubical arrangement of molecules at distance $e$ apart, then the number of molecules per unit area is $1 / e^{2}$, and hence the number of atoms $A_{1}$ per unit area is $n_{1} \frac{{ }^{\frac{2}{3}}}{e} e^{2}$. Let $m_{1}, m_{2}$, and so on be the masses of the atoms, and $v_{1}, v_{2}$ their mean velocities, then the collisional pressure per unit area due to the atoms $A_{1}$ is $n_{1}{ }^{\frac{2}{3}} m_{1} v_{1}^{2} / e^{2} \alpha_{1} 3$, if $\alpha_{1}$ is the full swing of the atom in its domain. For the total collisional pressure we have the sum of such pressures for the different sorts of atoms. For the pressure due to molecular attraction it does not matter whether we consider the attractions of the atoms separately or of the entire molecules; if we take the entire molecules, then we get the former expression $\Sigma r \phi(r) / 6 e^{3}$. For a solid compound submitted to hydrostatic pressure $p$ we have the equation

$$
\frac{1}{3 e^{2}}\left(\frac{n_{1}^{\frac{2}{3}} m_{1} v_{1}^{2}}{\alpha_{1}}+\frac{n_{2^{\frac{2}{3}}} m_{2} v_{2}^{2}}{\alpha_{2}}+\ldots\right)-p-\frac{1}{6 e^{3}} \Sigma r \phi(r)=0 .
$$

Kinetically, then, a compound in the solid state behaves like a mechanical mixture of its component atoms, because the molecules are almost as close to one another as the atoms in the molecule. It is evident that a complete kinetic theory of compounds must be a very complicated affair.

10. The Parameter of Molecular Force.-Hitherto we have managed to dispense with a close knowledge of the molecular attraction because we have always managed to eliminate it by using the fact that when the external pressure is small we can put

$$
\Sigma r \phi(r) / 6 e^{3}=2 \mathrm{D} / 3 e^{2}(e-\mathrm{E}),
$$

which is the fundamental equation for a solid free from external force. But it is desirable to obtain an independent estimate of molecular force for comparison with that given by this equation, and Quincke's measurements of the capillary tensions of the metals at their melting-points give an excellent opportunity (Pogg. Ann. cxxxv. and cxxxviii.).

I have shown (Phil. Mag. 5th ser. vol. xxvii.) that if the law of molecular force is $3 \AA \mathrm{m}^{2} / r^{4}$ then the surface-tension is proportional to $A m^{\frac{3}{3}} \rho^{\frac{5}{3}}$. Let us denote the surface-tension by 
$s$, then $A$ the parameter of molecular force is proportional to $s / m^{\frac{1}{2}} \rho^{\frac{3}{3}}$. But if $\phi(r)=3 \mathrm{~A} m^{2} / r^{4}$, then

$$
\Sigma r \phi(r)=12 \mathrm{~A} m \pi \rho \log \mathrm{R} / a,
$$

where $\log \mathrm{R} / a$ is nearly the same for all bodies not excessively small or large; hence

$$
\Sigma r \phi(r) / 6 e^{3}=2 \mathrm{~A} \rho^{2} \pi \log \mathrm{R} / a=2 \mathrm{D} / 3 e^{2}(e-\mathrm{E})=2 \mathrm{~J} c m \rho / 21 \mathrm{mb},
$$

or $\mathrm{A}$ is proportional to $\mathrm{cm} / \mathrm{mb} \rho$, or, as $c m$ is constant, to $1 / m b \rho$. Thus $s / m^{\frac{3}{3}} \rho^{\frac{5}{3}}$ is proportional to $1 / m b$, or $b s m^{\frac{2}{3}} / \rho^{\frac{3}{3}}$ is constant It is to be noticed that while $\Sigma r \phi(r) / 6 e^{3}$ or $2 \mathrm{~J} c m \rho / 21 \mathrm{mb}$ is independent of the molecular mass, the surface-tension $x \mathrm{~A} m^{\frac{1}{3}} \rho^{\frac{5}{3}}$ is not, so that if the molecular weights of the metals are not all the same multiple of the atomic weights, exceptional metals will give exceptional values of $b s m^{\frac{2}{3}} / \rho^{\frac{2}{3}}$.

The following is a list of the values of $1000 \mathrm{bsm} / \rho^{\frac{3}{3}} / s$ being given in milligrammes per millimetre. Where Quincke found two values of $s$ by different methods, both are given. In the case of lead the surface-tension was determined by myself.

$\begin{array}{rccccccc}\text { Pt. } & \text { Pd. } & \text { Au. } & \text { Au. } & \text { Ag. } & \text { Ag. } & \text { Sn. } & \text { Pb. } \\ \text { s...............169 } & 136 & 100 & 131 & 43 & 80 & 60 & \mathbf{3 4} \\ 1000 b s m^{\frac{2}{3}} / \rho^{\frac{2}{3}} \ldots .7 \cdot 3 & 7 \cdot 4 & 7 \cdot 4 & 9 \cdot 7 & 4 \cdot 0 & 7 \cdot 5 & 8 \cdot 7 & 6 \cdot 9 \\ & & & & & & & \\ \text { Zn. } & \text { Zn. } & \text { Cd. } & \text { Na. } & \text { K. } & \text { Hg. } & \text { Fe. } & \text { Fe. } \\ \text { s..............88 } & 83 & 71 & 26 & \mathbf{3 7} & \mathbf{5 9} & 102 & 97 \\ 1000 b s m^{\frac{2}{3}} / \rho^{\frac{2}{3}} \ldots .11 \cdot 4 & 10 \cdot 8 & 13 \cdot 6 & \mathbf{1 4} \cdot 4 & 26 & 28 & 4 \cdot 7 & \mathbf{4} \cdot 4\end{array}$

The value of the surface-tension of iron is uncertain, as is the case also with sodium and potassium ; for the value of $b$ for $\mathrm{Hg}, \mathrm{Na}$, and $\mathrm{K}, \mathrm{I}$ used the relation $b \mathrm{~T} m^{\frac{2}{s}}=\cdot 044$. The mean value of $1000 \mathrm{sm}^{\frac{2}{3}} / \rho^{\frac{2}{5}}$ for $\mathrm{Pt}, \mathrm{Pd}, \mathrm{Au}, \mathrm{Ag}, \mathrm{Sn}$, and $\mathrm{Pb}$ is $7 \cdot 4$, for $\mathrm{Zn}$ and $\mathrm{Cd}$ it is $12 \cdot 3$, which is once and a half as large or about $2^{\frac{2}{3}}$ as large, for $\mathrm{Na}$ it is twice as large, and for $\mathrm{Hg}$ and $\mathrm{K}$ four times. These relations and the whole subject of surface-tension in melted solids require further investigation. I have touched on the matter here to show that in the kinetic theory unfolded, the law of molecular force has not been lost sight of.

There are many phenomena of solids that invite investigation by the light of a kinetic theory, but it has seemed better to me to confine the present paper to the broad fundamental ones. One of the results of the inquiry is that the molecules of solids so nearly fill up their domains that the phenomena 
of crystalline form are the outward results of the form of the molecule; this has often been imagined to be the case, but the kinetic theory proves it. It is hardly necessary to enumerate the paths of experimental and theoretical investigation opened by a kinetic theory of solids; but it is obvious that a kinetic theory of liquids requires to be seen to.

Melbourne, April 1891.

LXIV. Note supplementary to a Paper on the Solitary Wave. By J. McCowan, M.A., B.Sc.*

T the concluding section of a paper "On the Solitary Wave," printed in the July number of the Philosophical Magazine, I offered a brief criticism of certain views of Sir George Strokes, which are contained in a paper ' On the Theory of Oscillatory Waves,' republished in the first volume of his Collected Papers, on the possibility of a solitary wave being propagated without change of form. He has, however, privately called my attention to the fact that not only had he himself seen the error of his former opinion, but had, in a paper "On the Highest Wave of Uniform Propagation" (Proc. (amb. Phil. Soc. vol. iv.), suggested a method by which the solitary wave might be approximated to by a sort of trial and error process, though he had not attempted the approximation itself, which would be laborious. He has also published a note to this effect in the September number of the Philosophical Magazine, which, however, I have only just seen, owing to my absence from town during the summer recess. I greatly regret that, in ignorance of his more recent paper, I should have offered any criticism of Prof. Stokes's earlier views, a criticism to which I was only led by the consideration that it would be impossible to pass over in silence the opinions of an authority of such eminence when they were in conflict with the results of my paper.

In his last "Note on the Theory of the Solitary Wave," already referred to, Prof. Stokes has explained how he had previously been led to a wrong conclusion, and this need not, I think, call for any remark on my part; but he has further offered certain objections to my criticism to which it is necessary for me to reply to prevent misinterpretation of my remarks, which were possibly too brief to be quite free from ambiguity. I refer to the third paragraph of the "Note." He begins by saying that he cannot agree with me that the expansion which he used is inadmissible. With respect to

* Communicated by the Author. 